\title{
Size-Dependent Dynamic Behavior of a Microcantilever Plate
}

\author{
Xiaoming Wang and Fei Wang \\ School of Mechanical and Electronic Engineering, Harbin Institute of Technology, Harbin 150001, China \\ Correspondence should be addressed to Fei Wang, wangfeivc@gmail.com
}

Received 18 February 2012; Revised 10 May 2012; Accepted 16 May 2012

Academic Editor: Grégory Guisbiers

Copyright ( $\odot 2012$ X. Wang and F. Wang. This is an open access article distributed under the Creative Commons Attribution License, which permits unrestricted use, distribution, and reproduction in any medium, provided the original work is properly cited.

\begin{abstract}
Material length scale considerably affects the mechanical properties of microcantilever components. Recently, cantilever-plate-like structures have been commonly used, whereas the lack of studies on their size effects constrains the design, testing, and application of these structures. We have studied the size-dependent dynamic behavior of a cantilever plate based on a modified couple stress theory and the differential quadrature method in this note. The numerical solutions of microcantilever plate equation involving the size effect have been presented. We have also analyzed the bending and vibration of the microcantilever plates considering the size effect and discussed the dependence of the size effect on their geometric dimensions. The results have shown that (1) the mechanical characteristics of the cantilever plate show obvious size effects; as a result, the bending deflection of a microcantilever plate reduces whereas the natural frequency increases effectively and (2) for the plates with the same material, the size effect becomes more obvious when the plates are thinner.
\end{abstract}

The miniaturized cantilever device is one of the fastest developing and most efficient types of micro-nano devices; it is widely used in the chemistry, physical, and biological fields [1-3]. In these applications, the dimensions of the cantilever are typically on the order of microns or even sub-microns. And the performance of cantilever device depends on the accurate extract of static bending and resonant frequency. Using test measurements and theoretical studies, scholars have recently proved that the mechanical properties of miniaturized structures critically depend on material lengthscale. The size effect shown in the mechanical properties considerably affects the static bending and resonant frequency shift of cantilever devices [4-6]. Therefore, studying the sizedependent behavior of cantilever devices is important.

Couple stress theory is an effective method for studying the size effect $[7,8]$. By rotating the gradient, the theory introduces material length-scale parameters to the constitutive equation. Lam et al. [9] proposed a modified couple stress model in which only one material length parameter is needed to capture the size effect, and the model has attracted many researchers in the past years. Kong et al. [10] used this theory to study the size effect of the EulerBernoulli beam natural frequency. Ma et al. [11] then studied the Timoshenko size effect that accounts for axial deformation and the Poisson effect.

Most widely known miniaturized devices are typical cantilever plate structures. Compared with a cantilever beam, a cantilever plate with a large surface area has higher sensitivity. However, current studies focus only on microbeam structures, and few studies on the size effect of cantilever plates have been reported. Tsiatas [12] developed a new Kirchhoff plate model for the static analysis of isotropic microplates with arbitrary shape based on a modified couple stress theory. Yin et al. [13] proposed a non-classical Kirchhoff plate model for the dynamic analysis of microscale. However, they have not studied the cantilever plate.

In this letter, we use the modified couple stress theory and the differential quadrature (DQ) method to study the size effect of a microcantilever plate. The characteristic length effect on static bending deflection and natural frequency of plate under uniform loads is analyzed. Furthermore, we discuss the dependence of the size effect on the geometric dimensions of the plate.

We study a rectangular Kirchhoff cantilever plate and assuming that the initial state of the microplate is flat and thin. The plate is subjected to a uniform distributed 
transverse load $q$ and hence undergoes a three-dimensional deformation state. The $w$ represents the deflection of the $z$ direction.

Based on the modified couple stress theory, the governing equation of the static bending of a microcantilever plate is [12]

$$
\left(D+D^{\prime}\right)\left(\frac{\partial^{4} w(x, y)}{\partial x^{4}}+2 \frac{\partial^{4} w(x, y)}{\partial x^{2} \partial y^{2}}+\frac{\partial^{4} w(x, y)}{\partial y^{4}}\right)=q(x, y)
$$

where $D$ is the classical bending rigidity of the plate and $D^{\prime}$ is the contribution of rotation gradients to the bending rigidity. The two parameters can be expressed as

$$
D=\frac{E h^{3}}{12\left(1-v^{2}\right)}, \quad D^{\prime}=\frac{E l^{2} h}{2(1+v)},
$$

where $E$ is Young's modulus, $v$ is Poisson's ratio, and $l$ denotes the material length-scale parameter representing the size effect, usually in the micro-nano order.

On the basis of (2), the governing equation for the vibration of the plate under uniform loads can be expressed as

$$
\begin{aligned}
\left(D+D^{\prime}\right) & \left(\frac{\partial^{4} w(x, y, t)}{\partial x^{4}}+2 \frac{\partial^{4} w(x, y, t)}{\partial x^{2} \partial y^{2}}+\frac{\partial^{4} w(x, y, t)}{\partial y^{4}}\right) \\
& +\rho h \frac{\partial^{2} w(x, y, t)}{\partial t^{2}}=q(x, y),
\end{aligned}
$$

where $\rho$ is the plate density and $h$ is the thickness of microcantilever.

The equation based on the modified couple stress theory contains only one material length-scale parameter. Therefore, the size effect can be well characterized. Meanwhile, (2) shows that when $l$ is zero, the equation degenerates to the classical equations of the Kirchhoff plate. It should be noted, in this letter we focus on the size effect and therefore ignored surface effect and induce residual stress.

For solving the equation of microplate, the analytical method is well-known cumbersome, and with the change of boundary and loading conditions, the difficulty of solving a sharp increase even cannot solve. In commonly used finite difference method and finite element method, the solving regional needs to be divided into fairly large number of grid or node, leading to large amount of calculation. However, as an effective numerical approach to solving boundary value problems, the DQ method can get enough precision of the calculation results using a few grid points, and this is the most significant advantage in comparison to other methods. We obtain the numerical solutions of static bending and vibration equations using this method.

By setting $\xi=x / L, \eta=y / b(0<\xi<1,0<\eta<1)$, where $L$ and $b$ are the length and width of the plate, respectively. The coordinate dimensionless form of (1) can be expressed as

$$
\left(D+D^{\prime}\right)\left(\frac{1}{L^{4}} \frac{\partial^{4} w}{\partial \xi^{4}}+2 \frac{1}{L^{2} b^{2}} \frac{\partial^{4} w}{\partial \xi^{2} \partial \eta^{2}}+\frac{1}{b^{4}} \frac{\partial^{4} w}{\partial \eta^{4}}\right)=q(\xi, \eta) .
$$

In the DQ method, the value of function partial derivative at one point can be approximately to weighted summation of function value of all the nodes, and the $n$ order derivative of function $g(x)$ at node $x_{i}$ is expressed as [14]:

$$
g^{(n)}\left(x_{i}\right)=\sum_{j=1}^{N} C_{i j}^{(n)} g\left(x_{j}\right) \quad n=1,2, \ldots, N_{x}-1,
$$

where $C_{i j}(n)$ is the $n$-order weighting coefficient and $N$ is the number of nodes.

By substituting (5) into (4), we can obtain the microplate static bending equation in DQ form:

$$
\begin{aligned}
& {\left[\frac{1}{L^{4}} \sum_{k=1}^{N_{x}} C_{i k}^{(4)} w\left(\xi_{k}, \eta_{j}\right)+2 \frac{1}{L^{2} b^{2}} \sum_{k=1}^{N_{x}} \sum_{l=1}^{N_{y}} C_{i k}^{(2)} \bar{C}_{j l}^{(2)} w\left(\xi_{k}, \eta_{l}\right)\right.} \\
& \left.+\frac{1}{b^{4}} \sum_{l=1}^{N_{y}} \bar{C}_{j l}^{(4)} w\left(\xi_{i}, \eta_{l}\right)\right]=\frac{q\left(\xi_{i}, \eta_{j}\right)}{\left(D+D^{\prime}\right)},
\end{aligned}
$$

where $i=1,2, \ldots, N_{x}, j=1,2, \ldots, N_{y} . N_{x}$ and $N_{y}$ are the number of nodes along the $x$ and $y$ directions of the plate. When (6) is expressed in matrix form, the equation becomes

$$
C \cdot w=q
$$

where $C$ is the weight coefficient matrix, $w$ is the nodal displacement array, and $q$ is the load vector.

The boundary conditions of the cantilever plate are

$$
\begin{gathered}
\omega=0, \quad \frac{\partial w}{\partial \xi}=0 ; \quad \xi=0, \\
\frac{\partial^{2} w}{\partial \xi^{2}}+\nu \frac{\partial^{2} w}{\partial \eta^{2}}=0, \quad \frac{\partial^{3} w}{\partial \xi^{3}}+(2-\nu) \frac{\partial^{3} w}{\partial \xi \partial \eta^{2}}=0 ; \quad \xi=1, \\
\frac{\partial^{2} w}{\partial \eta^{2}}+\nu \frac{\partial^{2} w}{\partial \xi^{2}}=0, \quad \frac{\partial^{3} w}{\partial \eta^{3}}+(2-\nu) \frac{\partial^{3} w}{\partial \xi^{2} \partial \eta}=0 ; \quad \eta=0, \\
\frac{\partial^{2} w}{\partial \eta^{2}}+\nu \frac{\partial^{2} w}{\partial \xi^{2}}=0, \quad \frac{\partial^{3} w}{\partial \eta^{3}}+(2-\nu) \frac{\partial^{3} w}{\partial \xi^{2} \partial \eta}=0 ; \quad \eta=1 .
\end{gathered}
$$

We use the equation substitution method to processing boundary conditions. Based on this method, the DQ equations in (8) substituted the nodes equation on $i=1,2, N_{x}-$ $1, N_{x} ; j=1,2, N_{y}-1, N_{y}$ in (6). The correctional weight coefficient matrix $C^{\prime}$ is obtained. The boundary conditions are introduced into (7).

We can see that $C^{\prime}$ and $q$ are known matrixes in (7), and then we can obtain the nodal displacement array $w$ under uniform loads. Finally, we can obtain the full displacement fields using a higher-order Lagrange interpolation. 
Similarly, by transforming and arranging (3), the cantilever vibration equation in DQ form becomes

$$
\begin{aligned}
& {\left[\frac{1}{L^{4}} \sum_{k=1}^{N_{x}} C_{i k}^{(4)} W\left(\xi_{k}, \eta_{j}\right)+2 \frac{1}{L^{2} b^{2}} \sum_{k=1 l=1}^{N_{x}} \sum_{i k}^{N_{y}} C_{i k}^{(2)} \bar{C}_{j l}^{(2)} W\left(\xi_{k}, \eta_{l}\right)\right.} \\
& \left.\quad+\frac{1}{b^{4}} \sum_{l=1}^{N_{y}} \bar{C}_{j l}^{(4)} W\left(\xi_{i}, \eta_{l}\right)\right]-\frac{\rho h \omega^{2}}{\left(D+D^{\prime}\right)} W\left(\xi_{i}, \eta_{j}\right)=\frac{q\left(\xi_{i}, \eta_{j}\right)}{\left(D+D^{\prime}\right)},
\end{aligned}
$$

where $W(x, y)$ is the mode function and $\omega$ is the angular frequency. The matrix form of (8) is

$$
C \cdot W-\rho h \omega^{2} \cdot W=q .
$$

According to the approach in (7), the natural frequency of the cantilever vibration can be obtained. Because the uniform load does not affect the natural frequency of the plate, the obtained value of the natural frequency is the natural frequency of the free vibration of the plate.

We present the numerical solution of the mechanical equations of a cantilever plate by the DQ method, then we expect to bring value to further analyses of the size effects of cantilever plates.

We set both length $L$ and width $b$ as $50 \mu \mathrm{m}$, thickness $h$ as $5 \mu \mathrm{m}$, modulus of elasticity $E$ as $50 \mathrm{Gpa}$, density $\rho$ as $2700 \mathrm{~kg} / \mathrm{m}^{3}$, and uniform load $q$ as $1 \mathrm{~N} / \mu \mathrm{m}$. The $9 \times 9$ nodes were chosen for the grid point in solving regional, and node distributions are taken based on well-accepted GaussChebyshev-Lobatto points [14].

In order to verify the effectiveness of the DQ method, we present the comparison results of analytical and DQ solution for deflection on $y=25 \mu \mathrm{m}$, as shown in Table 1 . We can see that, compared with analytical solutions, the error of DQ solution is less than $1 \%$ at different position $x$. It shows the satisfactory accuracy with a few grid points and full performances the characteristics of little computation quantity and high precision.

This letter is the first to present an investigation of the size effect of a cantilever plate by numerical simulation. The following issues are analyzed: (1) the changes in the cantilever deflection and natural frequency of the plate with certain geometric dimensions caused by the size effect and (2) the influence of the geometric dimensions of a plate on the strength of the size effect.

Figure 1 is obtained using (7), when Poisson's ratio as 0.3 , a thickness as $5 \mu \mathrm{m}$, at different length-scale parameters, the bending deflection of the cantilever plate along the $x$ direction $(y=25 \mu \mathrm{m})$. Here, $l$ has values of $0,1.5,3$, and $4.5 \mu \mathrm{m}$. Figure 1 shows that when the length-scale parameter $l$ increases, the deflection of the plate considerably decreases at the same location. For $x=25 \mu \mathrm{m}$, when the length-scale parameter increases from 0 to $4.5 \mu \mathrm{m}$, the bending deflection decreases from 0.15 to $0.03 \mu \mathrm{m}-\mathrm{a}$ reduction of $80 \%$. This reduction indicates an obvious size effect. We emphasize that when the length-scale parameter is zero $(l=0)$, the bending deflection is the classical elasticity solution. Therefore, the data show that, in considering the influence of the size effect, the deflection of the plate is substantially reduced.
TABLE 1: The comparison of analytical and DQ solution for deflection on $y=25 \mu \mathrm{m}$.

\begin{tabular}{lcccc}
\hline & \multicolumn{4}{c}{ Static bending deflection $\left(10^{-8} \mathrm{~m}\right)$} \\
Position $x$ & $12.5 \mu \mathrm{m}$ & $25 \mu \mathrm{m}$ & $37.5 \mu \mathrm{m}$ & $50 \mu \mathrm{m}$ \\
\hline Analytical solution & 5.214 & 14.925 & 24.051 & 32.014 \\
DQ solution & 5.171 & 14.805 & 23.845 & 31.720 \\
Error \% & 0.827 & 0.809 & 0.858 & 0.919 \\
\hline
\end{tabular}

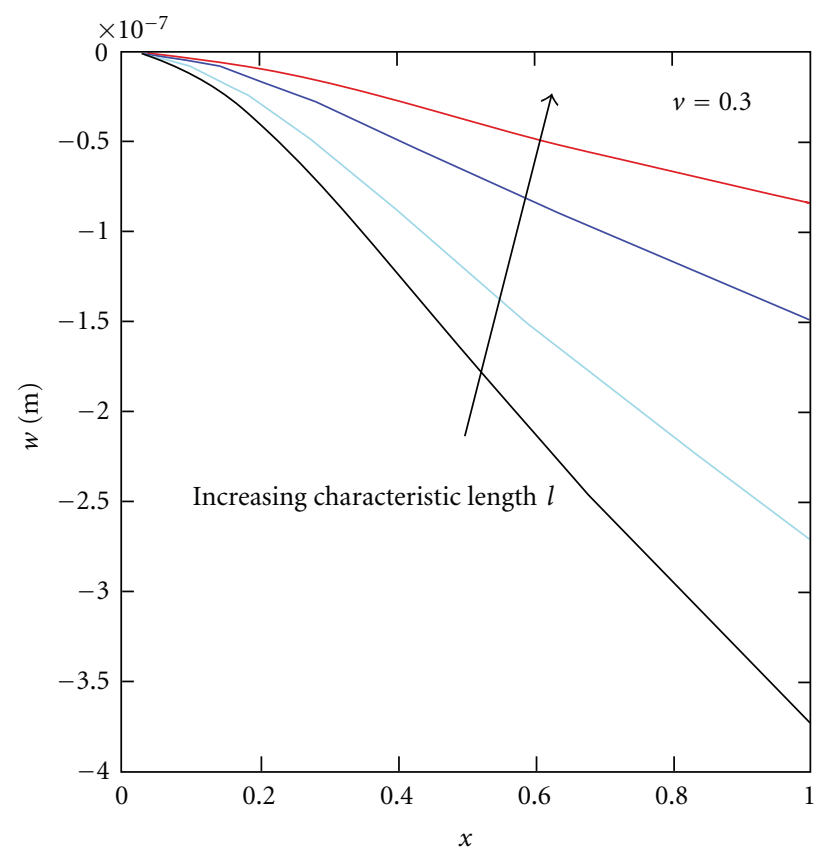

Figure 1: Deflection curve in the $x$ direction of a cantilever plate subjected to uniform loads; $l=0,1.5,3$, and $4.5 \mu \mathrm{m} ; \nu=0.3$.

Table 2 is obtained using (9), when having Poisson's ratio as 0.3 , a thickness as $5 \mu \mathrm{m}$, at different length-scale parameters, the first four orders of the natural frequencies of the cantilever plate; $l$ has values of $0,1.5,3$, and $4.5 \mu \mathrm{m}$. Table 1 shows that when the length-scale parameters increase, the natural frequency of each order mode considerably increases. For the first-order mode, when the length-scale parameters increase from 0 to $4.5 \mu \mathrm{m}$, the natural frequency increases from 14 to $30 \mathrm{MHz}$ - an increase of $114 \%$. Similarly, in considering the influence of the size effect, the natural frequency of the plate considerably increases.

Equation (2) can explain the phenomenon above, that is, when the length-scale parameters increases, the bending stiffness of the cantilever bending plate increases. Therefore, the plate bending deflection decreases and the natural frequency increases. In summary, when the length-scale parameters and plate thickness are in the same order of magnitude, the size effect cannot be disregarded. In addition, with the increase in length-scale parameters, the size effect becomes increasingly apparent. These results are in accordance with the size effect of microcantilever beams. 
TABLE 2: First four orders of the natural frequencies of the plate under uniform loads at different length-scale parameters.

\begin{tabular}{lcccc}
\hline \multirow{2}{*}{ Mode } & \multicolumn{4}{c}{ Natural frequency of vibration $\left(10^{8} \mathrm{~Hz}\right)$} \\
& $l=0 \mu \mathrm{m}$ & $l=1.5 \mu \mathrm{m}$ & $l=3 \mu \mathrm{m}$ & $l=4.5 \mu \mathrm{m}$ \\
\hline 1 & 0.14 & 0.17 & 0.23 & 0.30 \\
2 & 0.47 & 0.56 & 0.76 & 1.01 \\
3 & 0.69 & 0.81 & 1.10 & 1.45 \\
4 & 1.49 & 1.74 & 2.36 & 2.59 \\
\hline
\end{tabular}

To study the dependence of the size effect on the geometric dimensions of the cantilever plate, we set the definitions of the relative change in deflection as $\left(w-w_{0}\right) / w_{0}$ and the relative change in frequency as $\left(\omega-\omega_{0}\right) / \omega_{0}$, where $w_{0}$ and $\omega_{0}$ are respectively the bending deflection and natural frequency that do not consider the size effect $(l=0)$, and $w$ and $\omega$ are, respectively, the bending deflection and natural frequency that consider the size effect. By this definition, for the certain length-scale parameters, the influence of the size effect on mechanical properties is more substantial when relative change is greater.

The size effects of each point on the plate under the same conditions are the same. Thus, a fixed point $(x=25 \mu \mathrm{m}$, $y=25 \mu \mathrm{m})$ is chosen. Figure 2 and Table 3 show the same material (with $l=5 \mu \mathrm{m}$ ), and the relative changes in deflection and natural frequency with thickness and Poisson's ratios. The thickness is between 1 to $5 \mu \mathrm{m}$, and Poisson's ratios are $0.25,0.3$, and 0.35 .

We can see that, the size effect on mechanical properties weakens with the increase in thickness. For a Poisson's ratio of 0.3 , when the thickness of the cantilever plate increases from 1 to $5 \mu \mathrm{m}$, the relative change in deflection is reduced from 0.98 to 0.79 and the relative natural frequency decreases from 9.44 to 1.28 . These results indicate a considerably reduced size effect. In addition, for the cantilever plate of a certain thickness, the relative changes in deflection and natural frequency increase with the decrease in Poisson's ratio.

Based on the modified couple stress theory and the differential quadrature (DQ) method, we studied the sizedependent dynamic behavior of a rectangular cantilever plate. We provide numerical solutions to the static bending deflection and natural frequency and analyzed static bending deflection and the natural frequency vibration under the size effect and discussed the dependence of the size effect on the geometric dimensions of the plate. The results show that the DQ solution seems quite well to approach analytical solution and performances the satisfactory accuracy with a few grid points. When the length-scale parameters and the thickness are in the same magnitude, the size effect cannot be disregarded. Under the influence of the size effect, the bending deflection decreases and the natural frequency increases. For the cantilever plate with the same material, the size effect on the mechanical properties gradually increases when thickness decreases. This study provided data on the size effect of cantilever plates and serves as basis for future research.

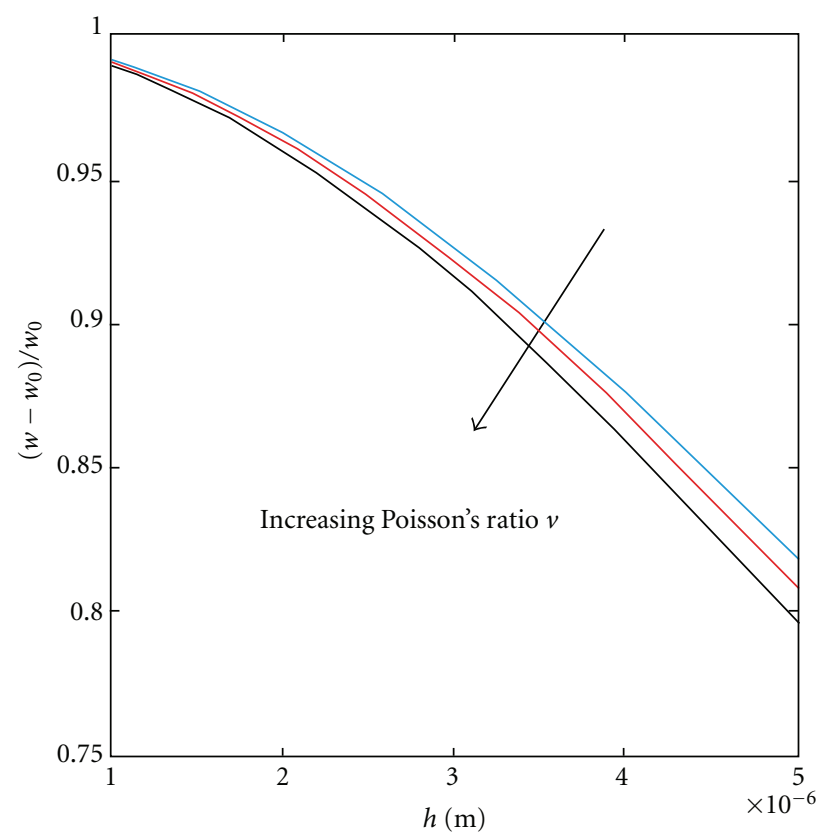

FIGURE 2: Relative change in the deflection of the plate with different thicknesses; $v=0.25,0.3,0.35$.

TABLE 3: Relative change in the first orders of the natural frequencies of the plate with different thicknesses.

\begin{tabular}{lcccc}
\hline \multirow{2}{*}{$\begin{array}{l}\text { Poisson's } \\
\text { ratio }\end{array}$} & \multicolumn{4}{c}{ Relative change in natural frequencies } \\
& $h=1 \mu \mathrm{m}$ & $h=2 \mu \mathrm{m}$ & $h=3.5 \mu \mathrm{m}$ & $h=5 \mu \mathrm{m}$ \\
\hline 0.25 & 9.63 & 4.39 & 2.20 & 1.34 \\
0.30 & 9.44 & 4.22 & 2.09 & 1.28 \\
0.35 & 8.90 & 4.03 & 1.99 & 1.20 \\
\hline
\end{tabular}

\section{Acknowledgment}

This work is supported by the National Natural Science Foundation of China Project no. 10902031 and China Postdoctoral Science Foundation Project no. 20090451001.

\section{References}

[1] F. Wang, J. Y. Chen, and X. Z. Zhao, "Comment on 'Mechanism of flexural resonance frequency shift of a piezoelectric microcantilever sensor during humidity detection," Applied Physics Letters, vol. 93, no. 9, Article ID 096101, 2008.

[2] D. Maraldo and R. Mutharasan, "Mass-change sensitivity of high-order mode of piezoelectric-excited millimeter-sized cantilever (PEMC) sensors: theory and experiments," Sensors and Actuators B, vol. 143, no. 2, pp. 731-739, 2010.

[3] F. Sansoz and T. Gang, "A force-matching method for quantitative hardness measurements by atomic force microscopy with diamond-tipped sapphire cantilevers," Ultramicroscopy, vol. 111, no. 1, pp. 11-19, 2010.

[4] S. Cuenot, C. Frétigny, S. Demoustier-Champagne, and B. Nysten, "Surface tension effect on the mechanical properties of nanomaterials measured by atomic force microscopy," Physical Review B, vol. 69, no. 16, Article ID 165410, p. 1, 2004. 
[5] L. G. Zhou and H. Huang, "Are surfaces elastically softer or stiffer?" Applied Physics Letters, vol. 84, no. 11, Article ID 1940, 3 pages, 2004.

[6] J. G. Guo and Y. P. Zhao, "The size-dependent elastic properties of nanofilm with surface effects," Journal of Applied Physics, vol. 98, Article ID 074306, 2005.

[7] Q. Ma and D. R. Clarke, "Size dependent hardness of silver single crystals," Journal of Materials Research, vol. 10, no. 4, pp. 853-863, 1995.

[8] W. J. Poole, M. F. Ashby, and N. A. Fleck, "Micro-hardness of annealed and work-hardened copper polycrystals," Scripta Metallurgica et Materialia, vol. 34, p. 559, 1996.

[9] D. C. C. Lam, F. Yang, A. C. M. Chong, J. Wang, and P. Tong, "Experiments and theory in strain gradient elasticity," Journal of the Mechanics and Physics of Solids, vol. 51, no. 8, pp. 1477$1508,2003$.

[10] S. Kong, S. Zhou, Z. Nie, and K. Wang, "The size-dependent natural frequency of Bernoulli-Euler micro-beams," International Journal of Engineering Science, vol. 46, no. 5, pp. 427437, 2008.

[11] H. M. Ma, X. L. Gao, and J. N. Reddy, "A microstructuredependent Timoshenko beam model based on a modified couple stress theory," Journal of the Mechanics and Physics of Solids, vol. 56, no. 12, pp. 3379-3391, 2008.

[12] G. C. Tsiatas, "A new Kirchhoff plate model based on a modified couple stress theory," International Journal of Solids and Structures, vol. 46, no. 13, pp. 2757-2764, 2009.

[13] L. Yin, Q. Qian, L. Wang, and W. Xia, "Vibration analysis of microscale plates based on modified couple stress theory," Acta Mechanica Solida Sinica, vol. 23, no. 5, pp. 386-393, 2010.

[14] T. Murmu and S. C. Pradhan, "Vibration analysis of nanoplates under uniaxial prestressed conditions via nonlocal elasticity," Journal of Applied Physics, vol. 106, no. 10, Article ID 104301, 2009. 

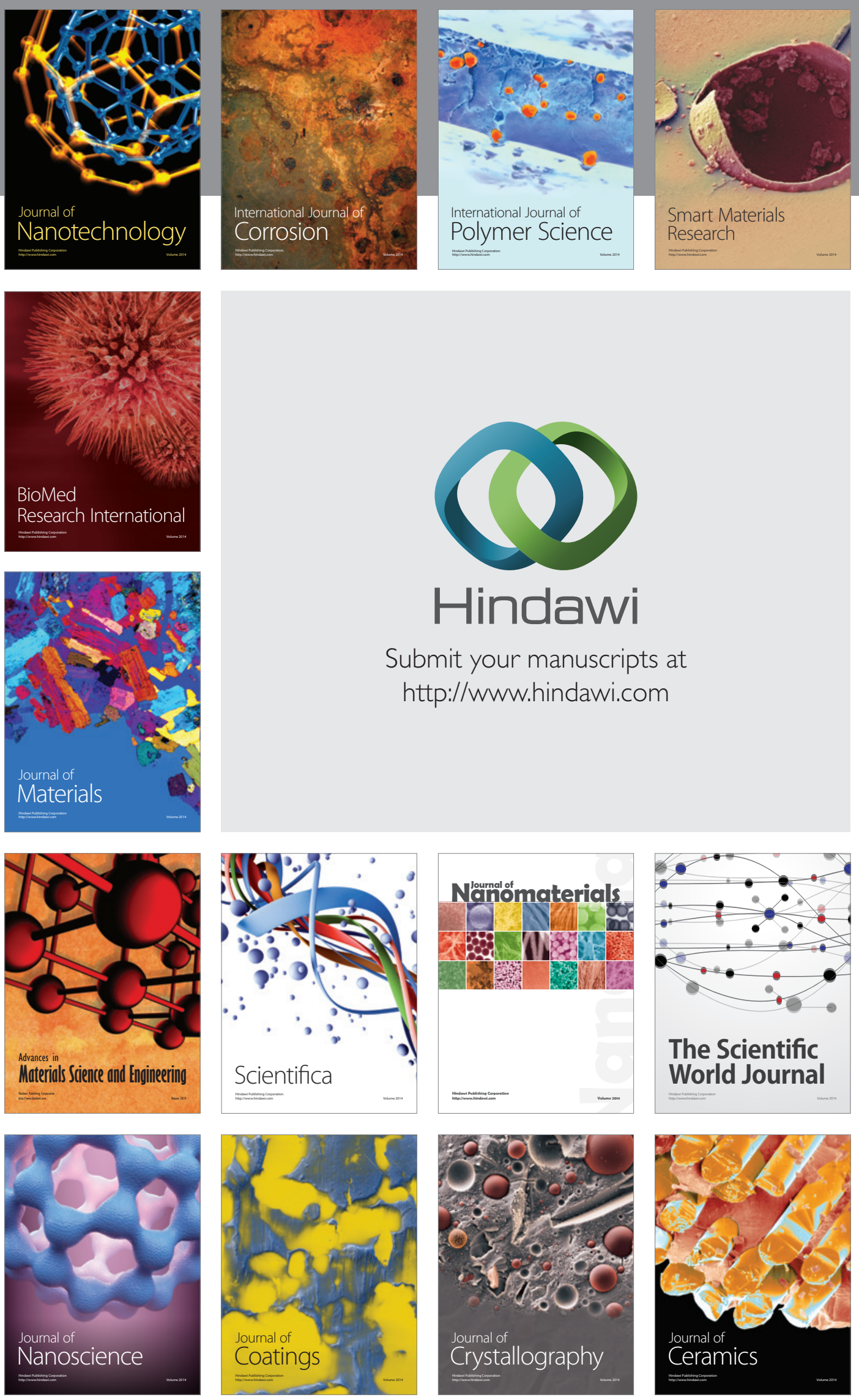

The Scientific World Journal

Submit your manuscripts at

http://www.hindawi.com

\section{World Journal}

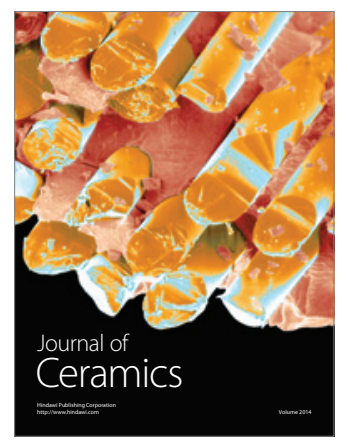

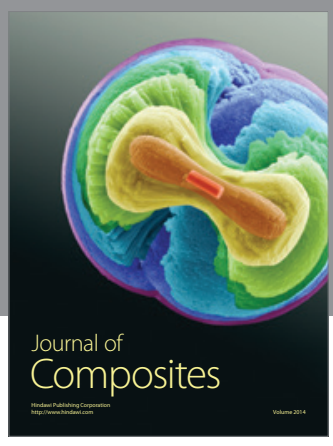
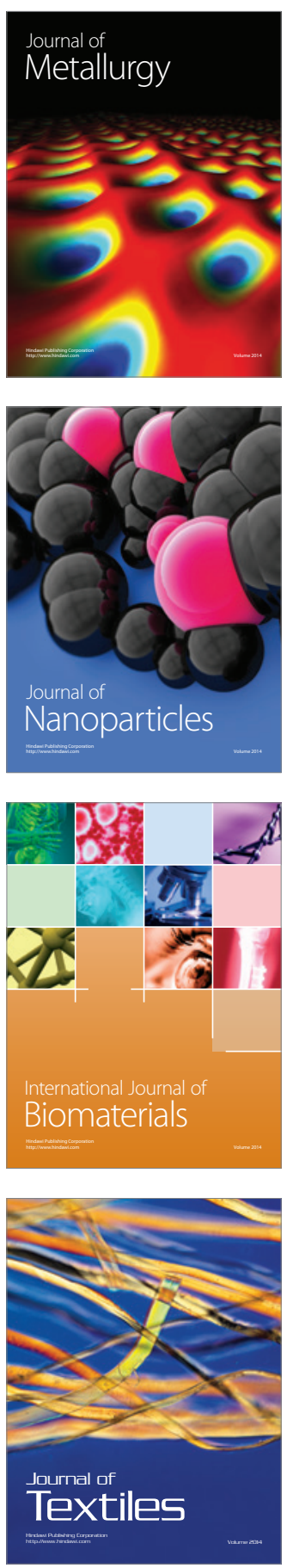